\title{
0 Impacto da Idade Materna Avançada sobre os Resultados da Gravidez
}

Impact of Advanced Maternal Age on the Outcome of Pregnancy

José G. Cecatti, Aníbal Faúndes

Fernanda G. C. Surita, Márcia M. A. Aquino

\section{RESUM0}

Objetivo: identificar o efeito da idade sobre os resultados maternos e perinatais das gestações ocorridas em mulheres com 40 anos ou mais.

Métodos: comparamos 494 gestantes com mais de 40 anos, com 988 gestantes com idade entre 20 e 29 anos, pareando-as por paridade. Após controlar possíveis variáveis confundidoras pela análise multivariada, a idade materna avançada manteve associação com a maior prevalência de hipertensão arterial, apresentação anômala, parto por cesária, hemorragia puerperal, índice de Apgar baixo, morte perinatal, natimortalidade e sofrimento fetal intraparto.

Resultados: a idade materna avançada esteve isoladamente associada à hipertensão arterial, apresentação anômala, diagnóstico de sofrimento fetal intraparto, parto por cesária e hemorragia puerperal. Com relação aos resultados neonatais, a idade materna avançada estava associada independentemente apenas ao baixo índice de Apgar, morte perinatal $e$ óbito fetal.

Conclusões: esses achados mostram a necessidade de assistência obstétrica adequada com atenção especial a esses fatores para procurar melhorar os resultados maternos $e$ perinatais das gestantes com idade avançada.

PALAVRAS-CHAVE: Idade materna. Gestação de alto risco.Complicações da gestação: Hipertensa. Mortalidade Perinatal. Óbito Fetal.

\footnotetext{
Departamento de Tocoginecologia

Disciplina de Obstetrícia

Faculdade de Ciências Médicas

Universidade Estadual de Campinas

Correspondência:

José Guilherme Cecatti

CAISM - UNICAMP

Caixa Postal 6030

13081-970 Campinas - SP, Brasil
} 
Introdução

São poucos os casos documentados de gravidez na sexta década de vida, que tenham evoluído até fases adiantadas da gravidez ou com recém-nascido viável ${ }^{8,12}$. No entanto, a gravidez na mulher entre os 40 e 45 anos é relatada na literatura médica e deve ser uma preocupação dos profissionais que dela cuidarão.

Algumas publicações têm denominado as mulheres com mais de 40 anos de "grávidas prémenopáusicas" ou grávidas "maduras" ${ }^{2,9}$ e isso já pode fornecer uma idéia sobre a baixa fertilidade nesse período. Realmente, há referência de um progressivo decréscimo da fertilidade com o aumento da idade nessa época da vida da mulher por uma diminuição paulatina da função ovariana, pela redução do número de oócitos e menor resposta às gonadotrofinas hipofisárias ${ }^{7,11,18}$.

A grande maioria das gestações ocorridas a partir dos 40 anos é de mulheres multíparas que engravidam, freqüentemente após decorrido longo período desde sua última gestação ou parto, período esse geralmente superior a $10 \operatorname{anos}^{2,17}$. Só mais recentemente, com o declínio da fecundidade das populações, é que a primeira gravidez em mulheres com idade mais elevada passou a constituir uma preocupação obstétrica também nos países em desenvolvimento. Entretanto, a primiparidade é a principal característica da gestação em mulheres com idade mais elevada nos países desenvolvidos ${ }^{5,14,15}$.

Há praticamente um consenso entre os autores sobre o pior prognóstico materno e perinatal da gestação em mulheres de 40 anos ou mais, quando comparadas a mulheres mais jovens ${ }^{3,5,10,16,19}$. Entretanto, quando se consideram os riscos associados à gravidez de mulheres idosas, é pertinente questionar se esses fatores são determinantes ou apenas acompanham a idade, e também a paridade, na ocorrência de piores resultados. No intuito de tentar esclarecer esse ponto, o presente estudo avaliou a influência da idade e de outros fatores sobre os resultados maternos e perinatais de gestações em mulheres com mais de 40 anos de idade.

\section{Pacientes e Métodos}

Após revisão das fichas obstétricas de 22.000 partos ocorridos na Maternidade do Departamento de Tocoginecologia da Universidade Estadual de
Campinas, no período de janeiro de 1977 a maio de 1990, foram identificados 509 casos de gestantes com 40 ou mais anos de idade, correspondendo a 2,31\% dos partos. Desse total, foram excluídos 15 casos por não preencherem os critérios de seleção previamente determinados: 8 por insuficiência de dados, 5 por apresentarem gestação gemelar e 2 por terem tido parto domiciliar.

Restaram para análise 494 casos de gestantes com idade avançada. Os parâmetros estudados foram comparados com os de 988 controles (gestantes com idade entre 20 e 29 anos), pareadas por paridade, que deram à luz no mesmo período e aleatoriamente selecionadas dentre os controles elegiveis, na proporção de dois controles para cada caso. Informações mais detalhadas sobre os métodos utilizados nesse estudo fazem parte de outra publicação ${ }^{4}$.

Foram estudadas as seguintes variáveis independentes e de controle: idade materna, estado marital, antecedente de hipertensão arterial, antecedente de cesárea, diabetes na gravidez, cardiopatia materna, infecção do trato urinário (ITU) na gravidez, número de consultas de prénatal (PN) e peso materno. As variáveis dependentes estudadas foram: hipertensão arterial, rotura prematura de membranas (RPM), tempo de rotura de membranas, apresentação fetal, tempo do período de dilatação, forma de parto, diagnóstico de sofrimento fetal intraparto (SFIP), hemorragia puerperal, idade gestacional (IG) e peso do recém-nascido (RN), índice de Apgar ao quinto minuto, malformações congênitas, mortalidade perinatal e adequação do peso para a idade gestacional (GIG - grande para a idade gestacional; PIG - pequeno para a idade gestacional).

Das fichas obstétricas foram colhidos os dados necessários para o estudo, que foram introduzidos num arquivo de dados, utilizando o programa Epi-Info. Após a depuração desses dados, procedeu-se a uma análise estatística, avaliando a distribuição percentual das categorias ou médias de cada variável dependente entre as mulheres dos dois grupos de idade, comparandose a significação das diferenças encontradas por meio do teste do $\chi^{2}$ ou t de Student. Por fim, foi efetuada uma análise multivariada por regressão logística. Para cada variável dependente principal construiu-se um modelo de regressão. As tabelas respectivas mostram os fatores preditores significativos para cada variável dependente, bem como seu respectivo fator de correlação [r] dentro do modelo. 


\section{Resultados}

A Tabela 1 apresenta um resumo dos resultados desfavoráveis das principais variáveis de controle segundo grupos de idade materna, mostrando diferenças significativas para o caso de antecedente de hipertensão, diabetes durante a gravidez, menor número de consultas de pré-natal e peso materno prévio à gestação superior ou igual a $70 \mathrm{~kg}$, todas mais freqüentes entre as idosas. $\mathrm{O}$ estado marital e o antecedente de cesárea foram semelhantes em ambos os grupos. Não houve diferença entre eles quanto ao tempo de rotura de membranas, quando se compararam suas médias, conforme mostra a Tabela 2 .

Tabela 1 - Resumo dos resultados das principais variáveis de controle segundo idade materna.

\begin{tabular}{|c|c|c|c|c|}
\hline \multirow{2}{*}{ Variáveis } & \multicolumn{2}{|c|}{ Idade materna } & \multirow[b]{2}{*}{$\chi^{2}$} & \multirow[b]{2}{*}{$\mathbf{p}$} \\
\hline & 40-49 anos & 20-29 anos & & \\
\hline Sem companheiro & 8,5 & 10,7 & 1,82 & NS \\
\hline Com antec, cesárea & 17,2 & 19,6 & 1,27 & NS \\
\hline Com antec, hipertensão & 35,2 & 14,4 & 85,34 & $<0,0001$ \\
\hline Com diabetes & 5,3 & 1,1 & 23,30 & $<0,0001$ \\
\hline Com cardiopatia & 4,1 & 2,8 & 1,55 & NS \\
\hline Com ITU & 10,3 & 12,2 & 1,07 & NS \\
\hline $0-5$ consultas pré-natal & 52,0 & 61,9 & 12,84 & $<0,0004$ \\
\hline Peso materno $>70 \mathrm{~kg}$, & 20,2 & 8,7 & 32,34 & $<0,0001$ \\
\hline
\end{tabular}

ITU - infecção do trato urinário

Tabela 2 - Média de tempo de rotura de membranas (em horas) segundo idade materna $\left(^{*}\right)$.

\begin{tabular}{lccccc} 
Idade materna & Média & DP & n & t & p \\
\hline $40-49$ anos & 4,5 & 12,5 & 482 & 0,7 & NS \\
$20-29$ anos & 4,0 & 11,7 & 962 & &
\end{tabular}

(*) Excluídos 38 casos sem dados.

A Tabela 3 mostra a via de parto nos grupos de idade materna. Para o total de gestantes, a cesárea foi mais de duas vezes mais freqüente no grupo de idosas, ocorrendo em 34,2\% dessas (diferença significativa). Entretanto, para avaliar a influência da via dos partos anteriores, a mesma análise foi realizada excluindo-se todos os casos e controles com antecedente de cesárea. Os resultados dessa análise, também expressos na mesma tabela, mostram que, ainda assim, a diferença permanece altamente significativa.

Tabela 3 - Distribuição porcentual da via de parto segundo idade materna e antecedente de cesárea.

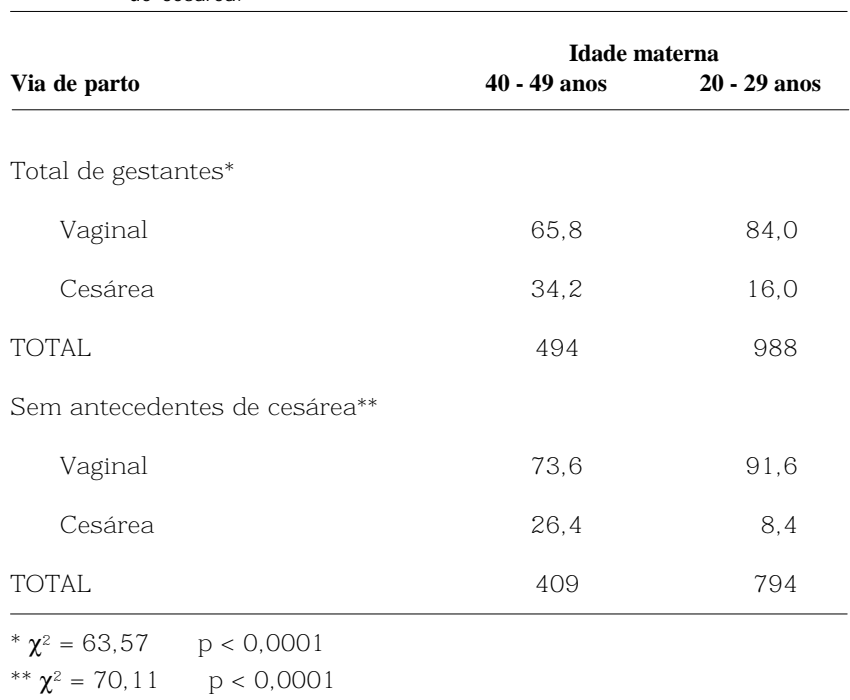

Não houve diferença estatisticamente significativa entre os grupos quando foram comparados segundo a média de idade gestacional do recém-nascido (Tabela 4) e do seu peso (Tabela 5). Quanto à condição perinatal, observou-se uma freqüência cerca de duas vezes maior de morte neonatal e quatro vezes maior de morte fetal tardia entre as gestantes com 40 anos ou mais (Tabela 6).

Tabela 4 - Média de idade gestacional do recém-nascido segundo idade materna (*).

\begin{tabular}{lccccr}
\hline Idade materna & Média & DP & $\mathbf{n}$ & $\mathbf{t}$ & $\mathbf{p}$ \\
\hline $40-49$ anos & 38,9 & 2,7 & 488 & 1,2 & NS \\
$20-29$ anos & 39,1 & 2,4 & 987 & & \\
\hline
\end{tabular}

(*) Excluídos 7 casos com idade gestacional ignorada.

Tabela 5 - Média do peso do recém-nascido segundo idade materna.

\begin{tabular}{lccccc}
\hline Idade materna & Média & DP & $\mathbf{n}$ & $\mathbf{t}$ & $\mathbf{p}$ \\
\hline & & & & & \\
$40-49$ anos & 3051,3 & 760,9 & 494 & 0,2 & NS \\
$20-29$ anos & 3041,5 & 617,1 & 988 & & \\
\hline
\end{tabular}


Tabela 6 - Distribuição porcentual da condição perinatal do recém-nascido até $07^{0}$ dia de vida segundo idade materna.

\begin{tabular}{lcc} 
& \multicolumn{3}{c}{ Idade materna } \\
Condição do RN & $\mathbf{4 0} \mathbf{- 4 9}$ anos & $\mathbf{2 0}-\mathbf{2 9}$ anos \\
\hline Nativivo & 91,9 & 97,6 \\
Natimorto & 5,3 & 1,2 \\
Morte neonatal & 2,8 & 1,2 \\
TOTAL & 494 & 988 \\
\hline
\end{tabular}

$\chi^{2}=27,08 \quad \mathrm{p}<0,0001$

Em razão da multiplicidade de fatores envolvidos na determinação dos resultados maternos e perinatais e também pela dificuldade de associá-los à idade materna, mesmo quando controlada pela principal variável confundidora, a paridade, procedeu-se à análise multivariada. Nela, cada modelo construído para cada variável dependente mostra quais condições das variáveis independentes (fatores) estiveram estatisticamente associadas.

Assim, a Tabela 7 mostra, por exemplo, que a idade materna avançada esteve isoladamente associada à hipertensão arterial, apresentação anômala, diagnóstico de sofrimento fetal intraparto, parto por cesárea e hemorragia puerperal. Com relação aos resultados neonatais, a Tabela 8 , por sua vez, mostra que a idade materna avançada estava associada independentemente apenas ao baixo indice de Apgar, morte perinatal e óbito fetal.

Tabela 7 - Fatores associados com as variáveis dependentes maternas e fetais, por análise multivariada.

\begin{tabular}{|c|c|c|c|c|}
\hline Variável & Fatores & $\chi^{2}$ & $\mathbf{p}$ & $\mathbf{r}$ \\
\hline $\begin{array}{l}\text { Hipertensão } \\
\text { arterial }\end{array}$ & $\begin{array}{l}\text { Antec. Hipertensão } \\
\text { Idade } \geq 40 \text { anos } \\
\text { Peso } \geq 70 \mathrm{~kg}\end{array}$ & $\begin{array}{r}280,22 \\
23,04 \\
12,86\end{array}$ & $\begin{array}{l}<0,0001 \\
<0,0001 \\
<0,0004\end{array}$ & $\begin{array}{l}0,466 \\
0,128 \\
0,092\end{array}$ \\
\hline $\begin{array}{l}\text { Tempo rotura } \\
\text { membr. }>12 \text { hs }\end{array}$ & Sem antec. cesárea & 5,44 & $<0,02$ & 0,071 \\
\hline RPM & Sem antec. cesárea & 4,84 & $<0,03$ & 0,053 \\
\hline $\begin{array}{l}\text { Apresentação } \\
\text { Não-cefálica }\end{array}$ & $\begin{array}{l}\text { Antec. cesárea } \\
\text { Idade } \geq 40 \text { anos }\end{array}$ & $\begin{array}{r}10,46 \\
6,72\end{array}$ & $\begin{array}{l}<0,002 \\
<0,01\end{array}$ & $\begin{array}{l}0,132 \\
0,099\end{array}$ \\
\hline $\begin{array}{l}\text { Per. dilatação } \\
\quad>12 \mathrm{hs}\end{array}$ & $\begin{array}{l}\text { Idade } \leq 29 \text { anos } \\
\text { Sem antec. cesárea }\end{array}$ & $\begin{array}{l}7,86 \\
4,83\end{array}$ & $\begin{array}{l}<0,006 \\
<0,03\end{array}$ & $\begin{array}{l}0,069 \\
0,048\end{array}$ \\
\hline SFIP & $\begin{array}{l}\text { Idade } \geq 40 \text { anos } \\
\text { Antec. hipertensão }\end{array}$ & $\begin{array}{l}17,01 \\
10,24\end{array}$ & $\begin{array}{l}<0,0001 \\
<0,002\end{array}$ & $\begin{array}{l}0,113 \\
0,084\end{array}$ \\
\hline Cesárea & $\begin{array}{l}\text { Antec. cesárea } \\
\text { Idade } \geq 40 \text { anos } \\
\text { Antec. hipertensão } \\
\text { Diabetes } \\
\text { Cons.pré-natal } \geq 6\end{array}$ & $\begin{array}{r}137,57 \\
37,23 \\
11,96 \\
8,05 \\
5,99\end{array}$ & $\begin{array}{l}<0,0001 \\
<0,0001 \\
<0,0006 \\
<0,005 \\
<0,02\end{array}$ & $\begin{array}{l}0,330 \\
0,168 \\
0,089 \\
0,070 \\
0,057\end{array}$ \\
\hline $\begin{array}{l}\text { Hemorragia } \\
\text { puerperal }\left({ }^{*}\right)\end{array}$ & Idade $\geq 40$ anos & 4,99 & $<0,03$ & 0,071 \\
\hline
\end{tabular}

(*) Excluída da análise a variável peso materno.
Tabela 8 - Fatores associados com as variáveis dependentes neonatais, por análise multivariada.

\begin{tabular}{|c|c|c|c|c|}
\hline Variável & Fatores & $\chi^{2}$ & $\mathbf{P}$ & $\mathbf{r}$ \\
\hline \multirow[t]{3}{*}{$\mathrm{IG}<37$ sem } & Cons. $\mathrm{PN}<6$ & 11,99 & $<0,0006$ & 0,115 \\
\hline & Antec. cesárea & 6,83 & $<0,01$ & 0,080 \\
\hline & Antec. hipertensão & 4,87 & $<0,03$ & 0,062 \\
\hline \multirow[t]{3}{*}{ Baixo peso } & Cons. $\mathrm{PN}<6$ & 19,56 & $<0,0001$ & 0,140 \\
\hline & Antec. hipertensão & 8,86 & $<0,003$ & 0,087 \\
\hline & Antec. cesárea & 3,94 & $<0,05$ & 0,046 \\
\hline \multirow[t]{3}{*}{ Apgar baixo } & Cons. $\mathrm{PN}<6$ & 6,75 & $<0,01$ & 0,096 \\
\hline & Antec. cesárea & 5,87 & $<0,02$ & 0,087 \\
\hline & Idade $\geq 40$ anos & 5,67 & $<0,02$ & 0,085 \\
\hline Malf.cong. & Cardiopatia & 6,19 & $<0,02$ & 0,116 \\
\hline \multirow[t]{4}{*}{ Morte perinatal } & Idade $\geq 40$ anos & 10,66 & $<0,002$ & 0,153 \\
\hline & Cons. $\overline{\mathrm{PN}}<6$ & 7,09 & $<0,008$ & 0,118 \\
\hline & Antec. hipertensão & 4,41 & $<0,04$ & 0,081 \\
\hline & Cardiopatia & 3,85 & $<0,05$ & 0,071 \\
\hline \multirow[t]{3}{*}{ Óbito fetal } & Idade $\geq 40$ anos & 11,72 & $<0,0007$ & 0,204 \\
\hline & Cons. $\overline{P N}<6$ & 7,24 & $<0,008$ & 0,150 \\
\hline & Cardiopatia & 4,52 & $<0,04$ & 0,104 \\
\hline \multirow[t]{2}{*}{ RN PIG } & Cons. $\mathrm{PN}<6$ & 13,88 & $<0,0003$ & 0,124 \\
\hline & Antec. hipertensão & 7,54 & $<0,0007$ & 0,085 \\
\hline \multirow[t]{2}{*}{ RN GIG } & Diabetes & 15,94 & $<0,0002$ & 0,130 \\
\hline & Peso $\geq 70 \mathrm{~kg}$ & 7,58 & $<0,006$ & 0,082 \\
\hline
\end{tabular}

\section{Discussão}

O propósito desse estudo foi o de avaliar os resultados maternos e perinatais das gestações ocorridas em mulheres com 40 ou mais anos de idade, tentando identificar o efeito isolado da idade pela exclusão de fatores confundidores que também se associam a esses resultados.

Depois de controlar o efeito de algumas variáveis potencialmente confundidoras, por meio da análise multivariada, a idade materna avançada manteve associação com maior ocorrência de hipertensão arterial, apresentação anômala, parto cesáreo, hemorragia puerperal, índice de Apgar baixo, morte perinatal, natimortalidade e sofrimento fetal intraparto.

Em relação à hipertensão arterial, a análise multivariada esclarece que ela está realmente associada à idade materna elevada. Foram identificados, contudo, outros fatores relacionados: o antecedente de hipertensão arterial e o peso materno maior ou igual a $70 \mathrm{~kg}$, o que é concordante com a literatura ${ }^{5,14,19}$. A falta de correlação entre diabetes e ocorrência de hipertensão arterial durante a gravidez, nessa análise, pode ser o reflexo da maior importância relativa do antecedente de hipertensão, idade avançada e maior peso, variáveis essas 
sabidamente associadas ao diabetes.

Da mesma forma que mostra a literatura, a incidência de apresentações anômalas foi significativamente maior no grupo das mulheres idosas, mesmo excluindo-se um importante fator de confusão como a paridade ${ }^{5,14,15,19}$.

Na literatura médica tem sido relatada uma maior incidência de cesárea nas mulheres a partir dos 40 anos, quando comparadas com as mais jovens ${ }^{5,11,13,14,15,19}$. O mesmo foi encontrado nesse estudo e essa associação manteve-se mesmo após o controle de possíveis confundidores. Essa associação foi tão importante que apenas o antecedente de cesárea apresentou maior correlação que a idade avançada, na análise multivariada.

A análise também mostrou que a idade maior de 40 anos teve a mais alta correlação com o diagnóstico de sofrimento fetal intraparto, seguido pelo antecedente de hipertensão arterial. Mesmo que o diagnóstico de SFIP seja controverso entre os diversos estudos, pela multiplicidade de fatores envolvidos, sua importância deve residir na determinação da via de parto e resultado perinatal subseqüentes ${ }^{5}$.

Pela análise multivariada o único fator que se associou à hemorragia puerperal foi a idade avançada. O baixo valor do fator de correlação para essa associação leva a pensar que é provável a existência de outro fator relacionado mais à hemorragia no puerpério. Tal fator poderia, por exemplo, ser uma diferença na paridade, oculta dentro da categoria de cinco ou mais partos. No caso desse estudo, ao passo que as multíparas com menos de 30 anos tinham quase todas paridade 5 , no grupo de 40 anos ou mais havia mulheres com paridade bem mais elevada. Outros autores encontraram aumento na incidência de hemorragia no puerpério, por inércia uterina, quando a paridade se eleva acima de cinco $^{6}$ e também na idade materna avançada ${ }^{14,19}$.

Os presentes resultados, quanto à vitalidade do recém-nascido, medida pelo índice de Apgar ao quinto minuto de vida, mostraram que a associação entre resultados mais desfavoráveis para as mais idosas existe e não podem ser somente explicados pela maior incidência de patologias durante a gestação e de intercorrências durante o trabalho de parto, como citam alguns autores ${ }^{1}$, visto que tal associação se manteve depois de se controlar por todas as outras variáveis confundidoras. Mais recentemente, tem-se relatado um pior prognóstico perinatal para as idosas apenas com relação à prematuridade e baixo peso pr,14,15,19. $^{\text {. }}$

Diferentemente do que se encontra na literatura médica ${ }^{5}$, não se confirmou a correlação entre idade avançada e malformações congênitas do RN. Pôde-se ainda identificar a cardiopatia materna como único fator associado às malformações congênitas, aparentemente sem nenhuma explicação conhecida. Entretanto, o pequeno número de gestações estudadas não permite deduções conclusivas sobre fatores associados às malformações congênitas.

Esses resultados mostram que uma mulher com mais de 40 anos, sem qualquer patologia, terá maior risco de apresentar em sua gestação hipertensão arterial, apresentação anômala, sofrimento fetal intraparto, parto cesáreo, hemorragia puerperal, recém-nascido com Apgar baixo e óbito fetal tardio ou perinatal.

Isto mostra a necessidade de tais mulheres terem essas informações, o que depende de profissionais de saúde conscientes sobre o problema, para que as orientem e prestem um adequado acompanhamento pré-natal e assistência ao trabalho de parto, parto, puerpério e ao recém-nascido, podendo, assim, minimizar os efeitos deletérios da idade materna elevada sobre a mulher e o recém-nascido. Isso já é uma realidade em países desenvolvidos, onde os resultados permitem afirmar que a gravidez, trabalho de parto e parto não diferem significativamente entre mulheres idosas e jovens quando a assistência é adequada ${ }^{15}$.

\section{SUMMARY}

\begin{abstract}
Most authors agree on the negative impact of pregnancy in women with advanced maternal age on maternal and perinatal outcome. However, it is not usual to evaluate if some considered risk factors are only confounders because they are present in women over forty years. In order to identify the isolated effect of age on maternal and perinatal outcome of pregnancies in women over forty, 494 pregnancies from this age group were compared to 988 pregnancies among women aged 20 to 29 years, matched by parity. After controlling possible confounding variables through multivariate analysis, advanced maternal age maintained its association with a higher prevalence of hypertension, malpresentation, cesarean section, postpartum hemorrhage, low Apgar score, perinatal death, late fetal death and intrapartum fetal distress. These findings show the need for adequate obstetrical care with special attention to those factors in order to improve maternal and perinatal outcome of pregnancies in women with advanced age.
\end{abstract}

KEY WORDS: Maternal age. High risk pregnancy. Hipertension Fetal death. Perinatal outcome. 


\section{Referências}

1.Berkowitz GS, Skovron ML, Lapinski RH, Berkowitz RL. Delayed childbearing and the outcome of pregnancy. N Engl J Med 1990; 322: 659-64.

2.Bird CC, McElin TW. The premenopausal gravida: a study of 23 obstetrical patients age 45 and older. J Reprod Med 1971; 6: 223-5.

3.Caspi E, Lifshitz Y. Delivery at 40 years of age and over. Isr J Med Sci 1979; 15: 418-21.

4.Cecatti JG, Parpinelli MA, Barini R, Faúndes A. A gestação na mulher a partir dos 40 anos. Influência da idade e outras variáveis sobre os resultados maternos e perinatais. Rev Bras Med Ginecol Obstet 1992; 3: 171-81.

5.Edge V, Laros RK Jr. Pregnancy outcome in nulliparous women aged 35 or older. Am J Obstet Gynecol 1993; 168: 1881-4.

6.Faúndes A, Fanjul B, Henriquez G, Mora G, Tognola C. Influencia de la edad y de la paridad sobre algunos parámetros de morbilidad materna y sobre la morbi-mortalidad fetal. Rev Chil Obstet Ginecol 1972; 37: 6-14.

7.Hansen JP. Older maternal age and pregnancy outcome: a review of the literature. Obstet Gynecol Surv 1986; 41:726-42.

8.Helm P, Ovlisen B. Pregnancy in a 50 year old. Acta Obstet Ginecol Scand 1988; 67: 373-4.
9.Kirz DS, Dorchester W, Freeman RK. Advanced maternal age: the mature gravida. Am J Obstet Gynecol 1985; 152: 7-12.

10.Marsico S, Mancuso A, Pizzo A, Grioli MF. La gravidanza ed il parto nella primigravida attempata. Minerva Ginecol 1986; 38: 343-9.

11.Moron AF, Almeida PAM, Camano L. Gestação em mulheres com 40 anos ou mais: Análise de variáveis maternas e do recém-nascido. Rev Paul Med 1989; 107: 203-11.

12.Natter CE. Pregnancy after fifty. Obstet Gynecol 1964; 24: 641-3.

13.Peipert JF, Bracken MB. Maternal age: an independent risk factor for cesarean delivery. Obstet Gynecol 1993; 81: 200-5.

14.Prysak M, Lorenz RP, Kisly A. Pregnancy outcome in nulliparous women 35 years and older. Obstet Gynecol 1995; 85: 65-70.

15.Roberts CL, Algert CS, March LM. Delayed childbearing are there any risks? Med J Aust 1994; 160: 539-44.

16.Spellacy WN, Miller SJ, Winegar A. Pregnancy after 40 years of age. Obstet Gynecol 1986; 68:452-4.

17.Stanton EF. Pregnancy after forty-four. Am J Obstet Gynecol 1956; 71: 270-82.

18.Taysi K. Preconceptional counseling. Obstet Gynecol Clin North Am 1988; 15: 167-78.

19.Viegas OA, Leong WP, Ahmed S, Ratnam SS. Obstetrical outcome with increasing maternal age. J Biosoc Sci 1994; 26: 261-7. 